\title{
Crystal Structure and Magnetic Properties of new Eu-Pd-Sn Compounds
}

\author{
I. Čurlík ${ }^{a, *}$, F. Gastaldo ${ }^{b}$, M. Giovannini $^{b, c}$, A.M. Strydom ${ }^{d}$ And M. Reiffers ${ }^{a, e}$ \\ ${ }^{a}$ Faculty of Humanities and Natural Sciences, University of Prešov, 17. novembra 1, 08116 Prešov, Slovakia \\ ${ }^{b}$ Department of Chemistry, University of Genova, Via Dodecaneso 31, 16146 Genova, Italy \\ ${ }^{c}$ CNR-SPIN, Corso Ferdinando Maria Perrone 24, 16152, Genova, Italy \\ ${ }^{d}$ Highly Correlated Matter Research Group, Department of Physics, University of Johannesburg, \\ PO Box 524, Auckland Park 2006, South Africa \\ ${ }^{e}$ Institute of Experimental Physics, SAS, Watsonova 47, 04001 Košice, Slovakia
}

\begin{abstract}
We report on the synthesis, crystal structure determination, and magnetic susceptibility measurements of $\mathrm{Eu}_{3} \mathrm{Pd}_{2} \mathrm{Sn}_{2}, \mathrm{EuPd}_{2} \mathrm{Sn}_{4}$, and EuPdSn 2 . For all three compounds a divalent state of Eu ions was obtained from the fitting of the magnetic susceptibilities. At low temperatures $\mathrm{Eu}_{3} \mathrm{Pd}_{2} \mathrm{Sn}_{2}, \mathrm{EuPd}_{2} \mathrm{Sn}_{4}$, and $\mathrm{EuPdSn}_{2}$ order magnetically at 23,12 , and $13 \mathrm{~K}$, respectively.
\end{abstract}

DOI: 10.12693/APhysPolA.131.1003

PACS/topics: 71.27.+a, 75.30.Mb, 75.30.-m

\section{Introduction}

The lanthanide elements $\mathrm{Eu}$ and $\mathrm{Yb}$ can exist in intermetallic compounds in the trivalent or in the divalent state. In the case of $\mathrm{Yb}$ these two states are $\mathrm{Yb}^{3+}$ $\left(4 f^{13}\right) / \mathrm{Yb}^{2+}\left(4 f^{14}\right)$, and for $\mathrm{Eu}$ intermetallics $\mathrm{Eu}^{3+}$ $\left(4 f^{6}\right) / \mathrm{Eu}^{2+}\left(4 f^{7}\right)$. Moreover, a certain number of $\mathrm{Yb}$ and Eu compounds show strong correlation between electrons, due to hybridization of $f$-electrons and conduction electrons. Owing to this fact, ternary compounds of europium and ytterbium show a great variety of anomalous physical phenomena ranging from quantum phase transitions to absence of magnetic order due to magnetic frustration [1-4].

In a previous paper the isothermal section at $600^{\circ} \mathrm{C}$ of the $\mathrm{Yb}-\mathrm{Pd}-\mathrm{Sn}$ system was studied [5]. Among the various intermetallic compounds present in this system, the crystal structure of $\mathrm{Yb}_{3} \mathrm{Pd}_{2} \mathrm{Sn}_{2}$ was determined $a b$ initio and $\mathrm{YbPdSn}_{2}$ was confirmed to crystallize in the $\mathrm{MgCuAl}_{2}$-type $[5,6]$. In a systematic search for new ternary compounds in the analogous $\mathrm{Eu}-\mathrm{Pd}-\mathrm{Sn}$ system, we have discovered the compounds $\mathrm{Eu}_{3} \mathrm{Pd}_{2} \mathrm{Sn}_{2}$, EuPdSn 2 , and $\mathrm{EuPd}_{2} \mathrm{Sn}_{4}$. A comparison between crystal structures of $\mathrm{Eu}_{3} \mathrm{Pd}_{2} \mathrm{Sn}_{2}$ and $\mathrm{Yb}_{3} \mathrm{Pd}_{2} \mathrm{Sn}_{2}$ was made through their polyanionic networks [7].

In this paper we report on the crystal structure of EuPdSn 2 and $\mathrm{EuPd}_{2} \mathrm{Sn}_{4}$ and on magnetic data of the three compounds, including $\mathrm{Eu}_{3} \mathrm{Pd}_{2} \mathrm{Sn}_{2}$.

\section{Experimental details}

Polycrystalline samples of the new compounds $\mathrm{Eu}_{3} \mathrm{Pd}_{2} \mathrm{Sn}_{2}, \mathrm{EuPdSn}$, and $\mathrm{EuPd}_{2} \mathrm{Sn}_{4}$ have been prepared by weighing the stoichiometric amount of elements

*corresponding author; e-mail: ivan.curlik@unipo.sk with the following nominal purity (mass\%): Eu - 99.99 (pieces), Pd — 99.95 (foil) and Sn — 99.999 (bar). Due to Eu being prone to slow oxidation in air, all the elements were weighed in a controlled atmosphere in a glovebox and afterwards enclosed and sealed in a small tantalum crucible by arc welding under pure argon. The samples were melted in an induction furnace under a stream of pure argon. To ensure homogeneity during the melting, the crucible was shaken continuously. Samples were then annealed in a resistance furnace $\left(\mathrm{Eu}_{3} \mathrm{Pd}_{2} \mathrm{Sn}_{2}\right.$ three weeks at $600^{\circ} \mathrm{C}, \mathrm{EuPdSn}_{2}$ - two weeks at $650^{\circ} \mathrm{C}$, $\mathrm{EuPd}_{2} \mathrm{Sn}_{4}$ - two weeks at $600^{\circ} \mathrm{C}$ ) and finally quenched in cold water. The crystal structure was examined by Xray powder diffraction using the vertical diffractometer X'Pert MPD (Philips, Almelo, The Netherlands), with $\mathrm{Cu} K_{\alpha}$ radiation and electronic microscopy and quantitative electron probe microanalysis (EPMA). The magnetic measurements were performed by a PPMS Dynacool (Quantum Design) device. The magnetic properties for all the three $\mathrm{Eu}-\mathrm{Pd}-\mathrm{Sn}$ compounds were measured in the temperature range of $2-300 \mathrm{~K}$. The measurement in zero field cooling regime for $\operatorname{EuPdSn}_{2}(m=15 \mathrm{mg})$ and $\operatorname{EuPd}_{2} \mathrm{Sn}_{4}(m=18.3 \mathrm{mg})$ were performed in applied magnetic field $H=1000 \mathrm{Oe}$, whereas for $\mathrm{Eu}_{3} \mathrm{Pd}_{2} \mathrm{Sn}_{2}$ $(m=4 \mathrm{mg})$ an applied magnetic field of 200 Oe was used.

\section{Results and discussion}

The results of the crystal structures for the three compounds are presented in Table I. The three compounds crystallize in orthorhombic lattices. $\mathrm{Eu}_{3} \mathrm{Pd}_{2} \mathrm{Sn}_{2}$ [7] was found to be isotypic to $\mathrm{La}_{3} \mathrm{Ni}_{2} \mathrm{Ga}_{2}$-type (Pbcm space group), whereas XRD patterns of $\mathrm{EuPdSn}_{2}$ and $\mathrm{EuPd}_{2} \mathrm{Sn}_{4}$ were successfully indexed in this work within $\mathrm{MgCuAl}_{2}$-type ( $\mathrm{Cmcm}$ space group) and $\mathrm{NdRh}_{2} \mathrm{Sn}_{4}$-type (Pnma space group), respectively.The obtained magnetic results for $\mathrm{Eu}_{3} \mathrm{Pd}_{2} \mathrm{Sn}_{2}, \mathrm{EuPdSn}_{2}$, and $\mathrm{EuPd}_{2} \mathrm{Sn}_{4}$ are 
shown in Figs. 1-3, and summarized in Table II. Well above the characteristic ordering temperatures all the $\chi(T)$ dependences follow the modified Curie-Weiss (CW) law $\chi(T)=\chi_{0}+\frac{C}{T-\Theta_{P}}$. From the fitting of $1 / \chi(T)$ with the inverse modified $\mathrm{C}-\mathrm{W}$ function (see the insets of the corresponding figures) we obtained the values of temperature independent susceptibility $\chi_{0}$, paramagnetic Weiss temperature $\Theta_{P}$ and effective magnetic moment $\mu_{\text {eff }}$ shown in Table II. In all the three compounds the obtained values of $\mu_{\text {eff }}$ are ascribable to $\mathrm{Eu}^{2+}$, since this is the only magnetic element in the three studied compounds. The $\mu_{\text {eff }}$ are close to the theoretical $\mathrm{Eu}^{2+}$ freeion value $7.94 \mu_{\mathrm{B}}$, indicating that Eu ions are in the magnetic and stable-moment state for all the compounds. It is worth noting the very small values of $\chi_{0}$ indicating very low Pauli susceptibilities for the three compounds. The observed values of paramagnetic Weiss temperatures are small and negative in case of $\mathrm{Eu}_{3} \mathrm{Pd}_{2} \mathrm{Sn}_{2}$, whereas the $\Theta_{P}$ is positive in $\mathrm{EuPdSn}_{2}$ and $\mathrm{EuPd}_{2} \mathrm{Sn}_{4}$ pointing to ferromagnetic correlations in the latter two compounds.

Crystallographic data on the novel Eu compounds.

TABLE I

\begin{tabular}{c|c|c|c|c|c}
\hline \hline \multirow{2}{*}{ Compound } & \multirow{2}{*}{$\begin{array}{c}\text { Pearson symbol/ } \\
\text { prototype }\end{array}$} & \multicolumn{3}{|c|}{ Lattice parameters [nm] } & \multirow{2}{*}{ Ref. } \\
\cline { 3 - 5 } & $a P 28 / \mathrm{La}_{3} \mathrm{Ni}_{2} \mathrm{Ga}_{2}$ & $0.6033(5)$ & $0.8755(4)$ & $1.4108(7)$ & {$[7]$} \\
\hline $\mathrm{Eu}_{3} \mathrm{Pd}_{2} \mathrm{Sn}_{2}$ & $o$ & 0 & \\
$\mathrm{EuPdSn}_{2}$ & $o S 16 / \mathrm{MgCuAl}_{2}$ & $0.4451(4)$ & $1.1587(2)$ & $0.7455(2)$ & this \\
$\mathrm{EuPd}_{2} \mathrm{Sn}_{4}$ & $o P 28 / \mathrm{NdRh}_{2} \mathrm{Sn}_{4}$ & $1.8592(2)$ & $0.4592(2)$ & $0.7264(8)$ & work
\end{tabular}

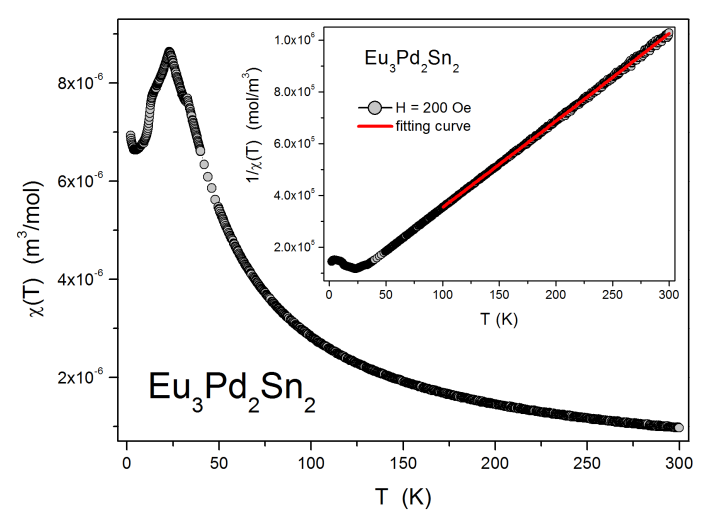

Fig. 1. Temperature dependence $\chi(T)$ for $\mathrm{Eu}_{3} \mathrm{Pd}_{2} \mathrm{Sn}_{2}$ measured in the temperature range $2-300 \mathrm{~K}$ in a magnetic field of $200 \mathrm{Oe}$. In the inset the $1 / \chi(T)$ dependence and fitting of the paramagnetic part as explained in the text is shown.

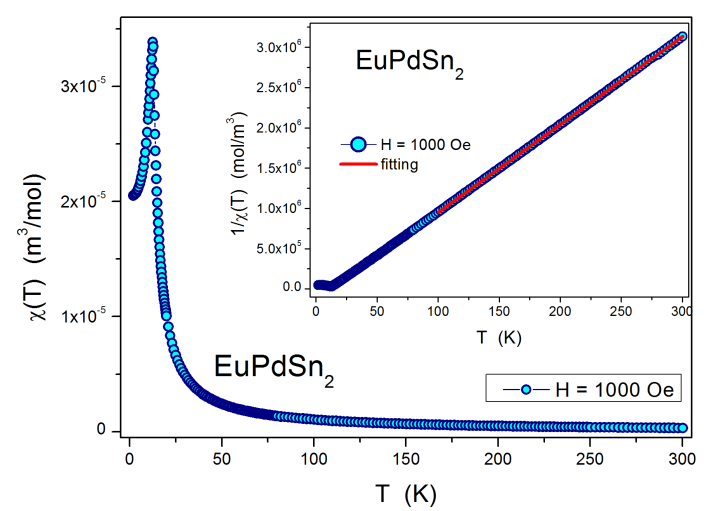

Fig. 2. Temperature dependence $\chi(T)$ for $\mathrm{EuPdSn}_{2}$ measured in the temperature range $2-300 \mathrm{~K}$ in a magnetic field of 1000 Oe. In the inset the $1 / \chi(T)$ dependence and a theoretical fit are shown.

At low temperatures, for all the studied compounds distinct cusps are observed in the magnetic susceptibilities $\chi(T)$ indicating that the compounds are magnetically

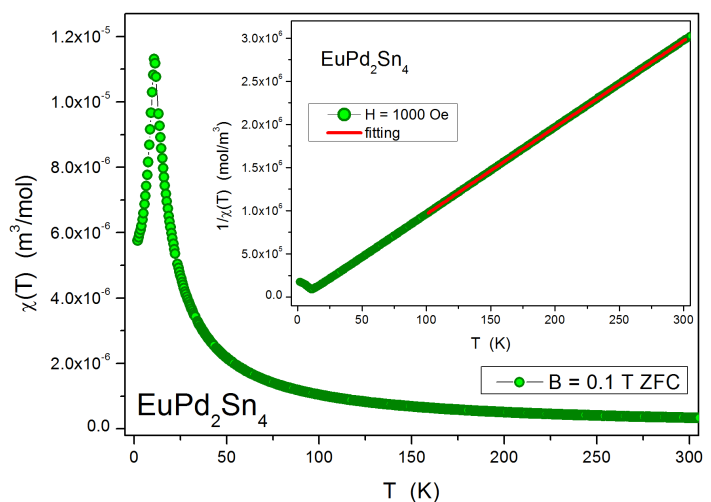

Fig. 3. Temperature dependence $\chi(T)$ for $\operatorname{EuPd}_{2} \mathrm{Sn}_{4}$ measured in the temperature range $2-300 \mathrm{~K}$ in magnetic field of 1000 Oe. In the inset the $1 / \chi(T)$ dependence together with a fit to extract magnetic parameters is shown.

TABLE II

Magnetic characteristics of the three $\mathrm{Eu}-\mathrm{Pd}-\mathrm{Sn}$ compounds $\left(T_{M}=\right.$ magnetic order temperature).

\begin{tabular}{c|c|c|c|c}
\hline \hline Compound & $\chi_{0}\left[\frac{\mathrm{m}^{3}}{\mathrm{~mol}}\right]$ & $\theta_{P}[\mathrm{~K}]$ & $\mu_{\mathrm{eff}} / \mathrm{Eu}\left[\mu_{\mathrm{B}}\right]$ & $T_{M}[\mathrm{~K}]$ \\
\hline $\mathrm{Eu}_{3} \mathrm{Pd}_{2} \mathrm{Sn}_{2}$ & $5 \times 10^{-9}$ & -5 & 7.95 & 23 \\
\hline $\mathrm{EuPdSn}_{2}$ & $4 \times 10^{-10}$ & 12.2 & 7.7 & 13 \\
\hline $\mathrm{EuPd}_{2} \mathrm{Sn}_{4}$ & $2.4 \times 10^{-10}$ & 4.5 & 7.95 & 12
\end{tabular}

ordered. A sharp cusp such as this in dc-magnetic susceptibility is indicative of long-range antiferromagnetic type ordering, although a more complex magnetic structure cannot be excluded (see e.g. [3]). In the case of $\operatorname{EuPdSn}_{2}$ and $\mathrm{EuPd}_{2} \mathrm{Sn}_{4}$ where positive Weiss temperature values were observed we note that the orthorhombic crystal structure of both structure types may plausibly have anisotropy in the magnetic exchange of the lanthanide ions, and that antiferromagnetic order at low temperature may be the outcome of one type of exchange dominating over another. Further measurements of magnetic, specific heat and transport properties are in progress in order to ascertain the nature of the ground state. 
The low-temperature magnetic behaviour of $\mathrm{Eu}_{3} \mathrm{Pd}_{2} \mathrm{Sn}_{2}$ (see Fig. 1) is characterized by the presence of a main cusp at $23 \mathrm{~K}$ with two shoulders at about $13 \mathrm{~K}$ and $33 \mathrm{~K}$ which might be due to spurious phases. In fact, the effect at $13 \mathrm{~K}$ is probably due to the presence in the compound of a minor phase of EuPdSn (as confirmed by XRD and EPMA analysis) which orders at $T_{N}=16 \mathrm{~K}[8]$.

\section{Conclusions}

The novel Eu compounds $\mathrm{Eu}_{3} \mathrm{Pd}_{2} \mathrm{Sn}_{2}, \mathrm{EuPdSn}_{2}$, and $\mathrm{EuPd}_{2} \mathrm{Sn}_{4}$ were synthesized and their crystal structures were derived from powder X-ray diffraction data. In the paramagnetic state the magnetic susceptibilities follow the Curie-Weiss behaviour with a divalent, magnetic state of Eu ions and very small Pauli susceptibilities. At low temperatures phase transitions to magnetically ordered states occur for all three compounds with ordering temperatures $T_{M}<23 \mathrm{~K}$. Further studies involving a full suite of physical properties are in progress on these compounds and will be reported in due course.

\section{Acknowledgments}

A.M.S. thanks the SA-NRF (93549), the FRC and URC of UJ for financial assistance. A.M.S. gratefully acknowledges the hospitality of Professor Giovannini during a visit to the University of Genova. This paper is the result of the Project implementation: University Science
Park TECHNICOM for Innovation Applications Supported by Knowledge Technology, ITMS: 26220220182, supported by the Research \& Development Operational Programme funded by the ERDF.

\section{References}

[1] M. Giovannini, I. Čurlík, F. Gastaldo, M. Reiffers, J.G. Sereni, J. Alloys Comp. 627, 20 (2015).

[2] I. Čurlík, M. Giovannini, J.G. Sereni, M. Zapotoková, S. Gabáni, M. Reiffers, Phys. Rev. B 90, 224409 (2014).

[3] K. Łątka, J. Przewoźnik, Yu. Verbovytskyy, A.P. Gonçalves, J. Alloys Comp. 650, 572 (2015).

[4] T. Muramatsu, T. Kanemasa, T. Kagayama, K. Shimizu, Y. Aoki, H. Sato, M. Giovannini, P. Bonville, V. Zlatic, I. Aviani, R. Khasanov, C. Rusu, A. Amato, K. Mydeen, M. Nicklas, H. Michor, E. Bauer, Phys. Rev. B 83, 180404 (R) (2011).

[5] M. Giovannini, R. Pasero, A. Saccone, Intermetallics 18, 429 (2010).

[6] F. Gastaldo, M. Giovannini, A.M. Strydom, R.F. Djoumessi, I. Čurlík, M. Reiffers, P. Solokha, A. Saccone, J. Alloys Comp. 694, 185 (2017).

[7] P. Solokha, I. Čurlík, M. Giovannini, N.R. Lee-Hone, M. Reiffers, D.H. Ryan, A. Saccone, J. Solid State Chem. 184, 2498 (2011).

[8] P. Lemoine, J.M. Cadogan, D.H. Ryan, M. Giovannini, J. Phys. Condens. Matter 24, 236004 (2012). 